language. Dyslexia is one of several distinct learning disabilities. It is a specific language-based disorder of constitutional origin, characterized by difficulties in single word decoding that reflect insufficient phonological processing. (See Millichap JG. Attention Deficit Hyperactivity and learning Disorders. PNB Publ, Revised 2001, for definitions and references to dyslexia, LD, and ADHD). Several methods of reading remediation have been reviewed by educators, including multisensory, modified alphabets, language-experience, individualized reading, synthetic phonics, linguistic decoding, and phonics systems (Millichap N, 1986). The multisensory approach to treatment of dyslexia is the most usual remediation employed by educators (Pavoni B, 2000).

The theory that dyslexia is due to an immaturity of cerebral function is supported by the pathological and neuroimaging evidence of developmental cerebral anomalies and electrophysiological studies. The anatomical location of the anomalies strongly supports the "phonological-linguistic," or deficient speech sound and decoding theory of dyslexia (Denckla MB, In Progress in Pediatric Neurology II. PNB Publ, 1994;p174). The left temporal-parietal area appears to be most critical in location of normal reading ability, but additional areas of the left hemisphere may be involved also. Some reports of acquired dyslexia following surgery on the brain have involved the left frontal lobe. A "disconnection theory" for dyslexia, involving impaired relays between the anterior and posterior areas of the left brain, has been proposed based on PET studies (Paulesu et al, 1996).

Dyslexia occurs in 5 to $10 \%$ of school children, at all levels of intelligence, from superior to low normal. Dyslexia may be an isolated abnormality or may be associated with other learning disabilities. Reading and spelling disability overlaps with ADHD and shows similar genetic characteristics but different brain localizations. Anatomically, left hemisphere deficits underly reading and other learning disabilities, whereas the right frontal lobe is involved in ADHD.

\title{
DEVELOPMENTAL RISKS OF INATTENTIVE BEHAVIOR
}

The developmental functioning, social, and environmental backgrounds of a community-based, epidemiological sample of 7-year-old children with pure inattentive behavior (I-subtype, $\mathrm{n}=31,1.3 \%$ of sample) were compared to that of children with pure overactive behavior (HI subtype, $\mathrm{n}=31$ ) ADHD-Combined type $(n=31)$ and a control group at the Maudsley Hospital, London, UK. A 2-item inattention subscale was derived from the Conners' (1969) questionnaire, namely "Fails to finish things" and "Inattentive, easily distracted." A cutoff score of 3 or more on these items was employed as the definition of inattentiveness. Other measures included parent and teacher interviews, general psychometric tests, Digit Span subtest of the WISC-R, Continuous performance task, paired-associates learning task, a Matching Familiar Figures Test, and objective measures of attentive behavior.

Inattentive behavior was significantly correlated with low self-esteem and need for repeated instructions in school, lower verbal IQ and general cognitive functioning, poor reading scores, and lower language related skills. Whereas the $\mathrm{HI}$ and Combined-ADHD groups showed more conduct and social-interaction problems, these outcomes were not encountered in the inattentive group. The fathers of inattentive children were more likely to have a low occupational status. (Warner-Rogers J, Taylor A, Taylor E, Sandberg S. laattentive behavior in childhood: Epidemiology and implications for development. Irnl of Learning Disabilities Nov-Dec 2000;33:520-536). (Respond: Dr Jody Warner-Rogers, Child Neuropsychiatry Clinic, Children's Outpatient Department, Maudsley Hospital, Denmark Hill, 
London SES 8AZ, England).

COMMENT. In this study conducted only in boys, inattentive behavior is a developmental risk factor for impairment of general cognitive functioning, poor reading and language skills, and a low self-esteem in school. In contrast to boys with hyperactivity and combined $\mathrm{ADHD}$, pure inattentive behavior is not associated with increased susceptibility to conduct problems or disturbed family relationships. Early recognition of ADD-inattentive subtype should allow prompt interventional treatment. Although boys outnumber girls with a $4: 1$ ratio for ADHD-HI subtype, the sex ratio is lower (2:1) for the ADHD-Inattentive type. Inattentiveness is relatively more prevalent among girls with ADHD, and any sexrelated cognitive and social risk factors would be of interest.

Parent-rated psychosocial correlates in preschool ADHD children.

In a study of 25 children with ADHD (21 males, 4 females; mean age 4.8 years), compared to 25 normal controls, at the IWK-Grace Health Centre, Halifax, Canada, parents rated their ADHD children as significantiy more aggressive, more demanding, less socially skilled, and less compliant. In contrast, the children perceived themselves as equally competent and as socially accepted as their peers. The parents viewed themselves as less competent parents. (DeWolfe NA, Byrne JM, Bawden HN. ADHD in preschool children: parent-rated psychosocial correlates. Dev Med Child Neurol Dec 2000;42:825-830). Presumably, these children had ADHDcombined type. No distinction of subtypes was made.

\section{MAGNETIC BRAIN STIMULATION IN ADHD}

Transcranial magnetic brain stimulation was performed in 27 children and adolescents, aged 4 to 18 years, with ADHD in the Services of Pediatric Neurology and Clinical Neurophysiology, Miguel Servet Hospital, Zaragoza, Spain. The motor evoked potential was recorded at the biceps brachii. Central motor conduction time mean value, calculated by cervical root stimulation, was very significantly increased $(\mathrm{P}<0.001)$ compared to normal controls. The finding reflects a delay in the maturation of the corticomotor-neuronal system. A side-to-side difference in the central motor conduction times in the ADHD group ( $\mathrm{P}=.03)$ suggests an hemispheric asymmetry in maturation. (Ucles P, Serrano J-L, Rosa F. Central conduction time of magnetic brain stimulation in attention-deficit hyperactivity disorder. I Child Neurol Nov 2000;15:723-728). (Respond: Dr Paulino Ucles, Almagro, 11 7-B, E-50004 Zaragoza, Spain).

COMMENT. Central motor conduction time in ADHD measured by transcranial magnetic brain stimulation may provide assistance in diagnosis and evidence supporting the theory of delayed cerebral maturation.

\section{SEIZURE DISORDERS}

\section{BEHAVIOR PROBLEMS ANTEDATING EPILEPSY ONSET}

The prevalence and nature of behavior problems among 224 children (ages 4 to 14 years) with epilepsy, in the six month period before the first recognized seizure, were studied at the Indiana School of Nursing, Indianapolis. Compared to their 135 healthy siblings, children with seizures had a higher than expected rate of antecedent behavior problems, with $32 \%$ in the clinical or at-risk range. Those with previous events suggestive of seizures had a $39 \%$ risk of behavior disorders before the first recognized seizure occurred. Children with seizures, especially 